\title{
Lung carcinoma after radiotherapy and chemotherapy for Hodgkin's disease
}

\author{
R. A. MATTHAY, S. K. ZORN, M. S. MITCHELL, AND R. J. PAPAC
}

From the Department of Medicine, Yale-New Haven Hospital and the West Haven Veterans Administration Hospital, Yale University School of Medicine, New Haven, Connecticut, USA

Matthay, R. A., Zorn, S. K., Mitchell, M. S., and Papac, R. J. (1977). Thorax, 32, 628-631. Lung carcinoma after radiotherapy and chemotherapy for Hodgkin's disease. Two patients, aged 29 and 40, with nodular sclerosing Hodgkin's disease, in complete remission for four and six years respectively after intensive radiotherapy and combination chemotherapy, developed carcinoma of the lung in the previous radiation port. Although radiotherapy and chemotherapy can eradicate Hodgkin's disease involving the thorax, this treatment may be associated with the development of a secondary pulmonary malignancy, particularly in a previous radiation port, and several years may elapse before the new tumour becomes evident.

Treatment of Hodgkin's disease with intensive radiotherapy ( $>3000$ rads) followed by combination chemotherapy has been associated with a $9 \cdot 7 \%$ incidence of second malignancies including acute leukaemia, carcinoma of the colon, squamous cell carcinoma of the skin, and fibrosarcoma of the chest wall (Canellos et al., 1975). We describe two cases of primary lung cancer occurring in a previous radiation port for Hodgkin's disease after remission had been obtained with intensive radiotherapy followed by combination chemotherapy.

\section{Case reports}

\section{CASE 1}

A 29-year-old man was admitted to hospital in 1975 for evaluation of haemoptysis and a right lung mass. In August 1964, he was treated with radiotherapy (4500 rads) to cervical, axillary, and mediastinal areas for nodular sclerosing Hodgkin's disease (Figs 1 and 2). He remained well until 1967 when he developed alcohol-induced abdominal pain. A lymphangiogram revealed abnormal abdominal lymph nodes, and radiotherapy (4000 rads) was administered to the abdomen. In 1971, he received six courses of COPP (cytoxan, vincristine, prednisone, and procarbazine) over six months for recurrent Hodgkin's disease manifested by fever, left axillary adenopathy, left pleural effusion, and bone lesions. He became asymptomatic, the adenopathy disappeared, and the chest radiograph cleared after this treatment.
However, in October 1975, the patient developed haemoptysis, and a chest radiograph demonstrated a mass in the right upper lobe (Fig. 3). The patient had never smoked cigarettes. Physical examination was normal, but on fibreoptic bronchoscopy a friable bleeding mass was noted occluding the right upper lobe, posterior segment. Biopsy through the bronchoscope showed tumour of undetermined aetiology. Thoracotomy revealed a poorly differentiated epidermoid carcinoma involving the right hilum and mediastinal lymph nodes. The patient was treated with cytoxan, methotrexate, and Corynebacterium parvum with some regression of the lung mass. $\mathrm{He}$ is alive and well 22 months later with a residual pulmonary mass on the chest radiograph.

\section{CASE 2}

A 41-year-old man was admitted to hospital in 1975 for evaluation of haemoptysis. In 1963, he was treated with radiotherapy (3500 rads) to cervical, axillary, and mediastinal areas for nodular sclerosing Hodgkin's disease localised to cervical and axillary lymph nodes. The patient remained well until 1966 when he was treated with 3400 rads to the pelvis and para-aortic lymph nodes for recurrent Hodgkin's disease in the femoral and inguinal lymph nodes. In 1969, he received cyclical chemotherapy (nitrogen mustard, vincristine, velban, procarbazine, and prednisone) for a recurrence manifested by night sweats and a middle lobe mass proven by open lung biopsy to be 


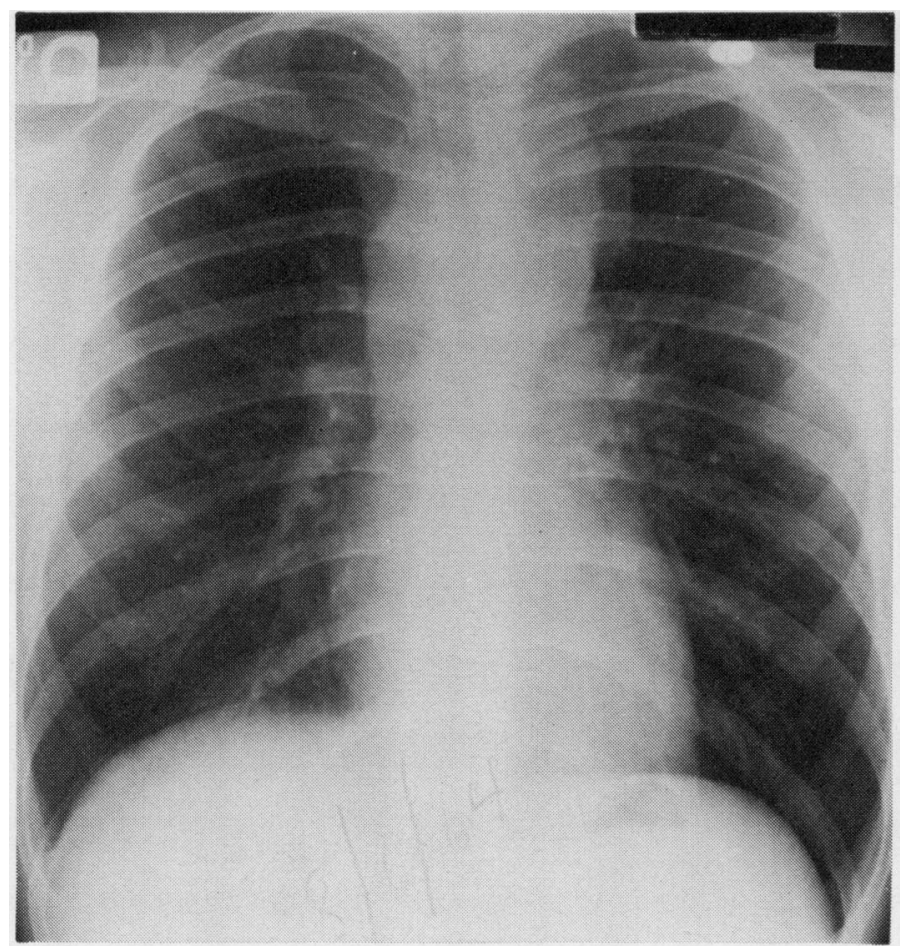

Fig. 1 Case 1. Posterior-anterior $(P A)$ chest radiograph in August 1964, demonstrating mediastinal widening due to thoracic involvement with nodular sclerosing Hodgkin's disease.

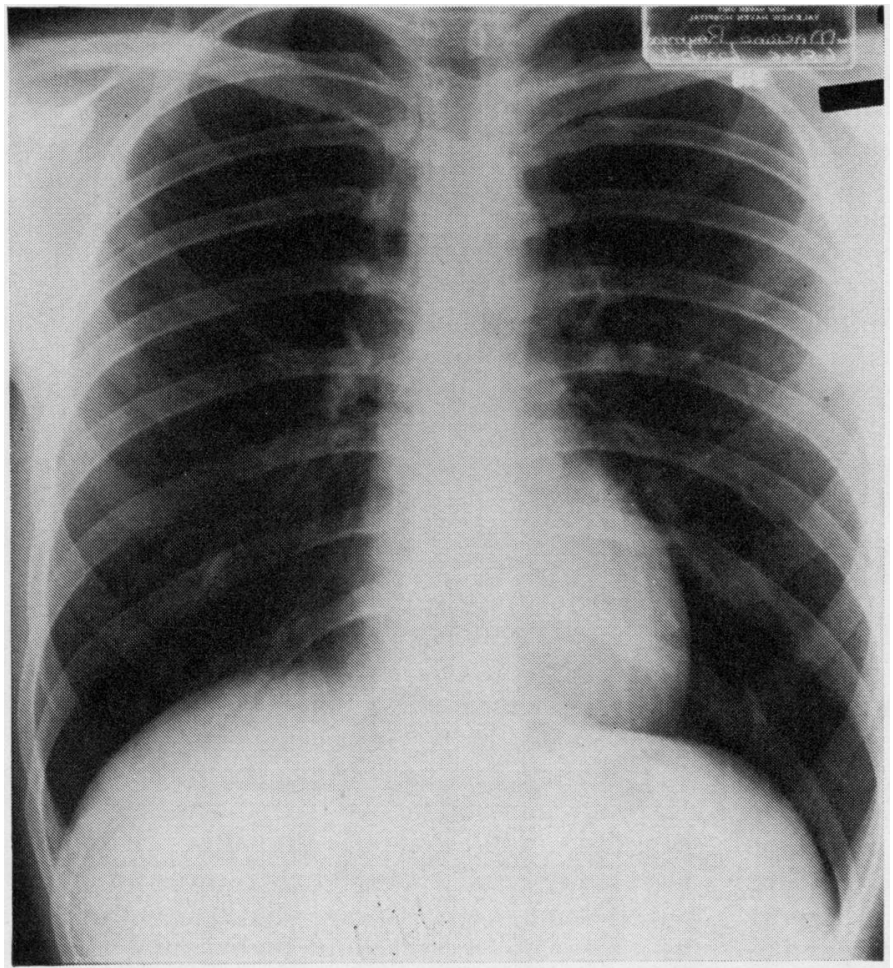

Fig. 2 Case 1. PA chest radiograph in June 1966 after thoracic irradiation shows a normal mediastinum. 


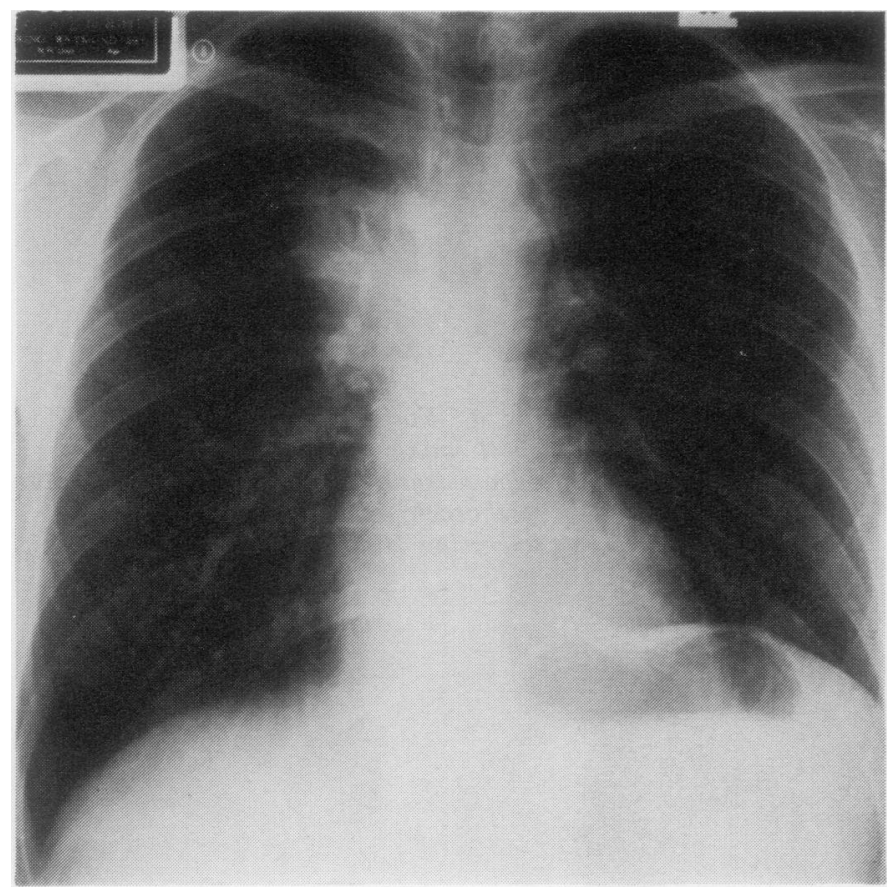

Fig. 3 Case 1. PA chest radiograph in October 1975 demonstrates a right upper lobe mass. Thoracotomy revealed a poorly differentiated epidermoid carcinoma.

nodular sclerosing Hodgkin's disease. The lung mass cleared and the patient remained in remission until 1975 when he noted haemoptysis. He had smoked 30 cigarettes daily for 20 years. Physical examination revealed crackles over both lungs. A chest radiograph showed diffuse interstitial infiltrates. Fibreoptic bronchoscopy revealed an endobronchial mass on the medial bronchial wall just above the left lower lobe and forceps biopsy of this lesion through the bronchoscope showed a poorly differentiated carcinoma. In spite of methotrexate and vincristine therapy the patient died four months later in respiratory failure. Necropsy revealed a poorly differentiated adenocarcinoma in the right lower lobe bronchus diffusely metastatic to both lungs with lymphatic and pericardial invasion. There was no evidence of any organ involvement with Hodgkin's disease at necropsy.

\section{Discussion}

The possibility of radiation and/or chemotherapy causing a second tumour can best be studied in patients surviving for prolonged periods after treatment for advanced malignant disease, such as Hodgkin's disease (Arseneau et al., 1972; Canellos et al., 1975; Schein and Winokur, 1975). Canellos and co-workers (1975) identified second tumours in six of 62 patients $(9 \cdot 7 \%)$ treated with radio- therapy and chemotherapy for Hodgkin's disease. However, patients in complete remission after intensive radiotherapy followed by a relapse before receiving combination chemotherapy had the highest risk of developing second malignancies18.5 times greater than expected when analysed according to age, sex, and man-years of follow-up (Canellos et al., 1975). In both patients in this series complete remission was obtained after intensive radiotherapy, and relapse of Hodgkin's disease occurred before the administration of combination chemotherapy.

Prior to this report primary lung cancer occurring in the radiation port of patients with Hodgkin's disease treated with both radiotherapy and chemotherapy has not been emphasised. Possible causes of these second lung tumours include immunosuppressive action of chemotherapy, derangement of nucleic acid synthesis due to chemotherapy, radiation-induced cell transformation, impairment of cellular immunity in Hodgkin's disease, and a combination of the above factors (Gross et al., 1969; Arseneau et al., 1972; Penn, 1974; Canellos et al., 1975; Penn, 1976). Also, cigarette smoking in our second case may have played a part. We conclude that although intensive radiotherapy and chemotherapy can lead to prolonged remission of Hodgkin's disease involving the thorax, a second lung malignancy may de- 
velop, particularly in a previous radiation port, and several years may elapse before the new tumour becomes evident.

\section{References}

Arseneau, J. C., Sponzo, R. W., Levin, D. L., Schnipper, L. E., Bonner, H., Young, R. C., Canellos, G. P., Johnson, R. E., and DeVita, V. T. (1972). Nonlymphomatous malignant tumcrs complicating Hodgkin's disease: possible association with intensive therapy. New England Journal of Medicine, 287, 1119-1122.

Canellos, G. P., DeVita, V. T., Arseneau, J. C., Whang-Peng, J., and Johnson, R. E. C. (1975). Second malignancies complicating Hodgkin's disease in remission. Lancet, 1, 947-949.

Gross, P., Pfitzer, E. A., Watson, J., DeTreville, R. T. P., Kaschak, M., Tolker, E. B., and Babyak,
M. A. (1969). Experimental carcinogenesis: bronchial intramural adenocarcinomas in rats from $x$-ray irradiation of the chest. Cancer, 23, 10461060.

Penn, I. (1974). Chemical immunosuppression and human cancer. Cancer, 34, 1474-1480.

Penn, I. (1976). Second malignant neoplasms ass $\approx$ ciated with immunosuppressive medications. Cancer, 37, 1024-1032.

Schein, P. S., and Winokur, S. H. (1975). Immunosuppressive and cytotoxic chemotherapy: long-term complications. Annals of Internal Medicine, 82, 84-95.

Requests for reprints to: Richard A. Matthay, MD, Pulmonary Division, Department of Medicine, Yale University School of Medicine, 333 Cedar Street, New Haven, Connecticut 06510, USA. 